pite consistently high serum osmolality, but he maintained an intact ADH response to nausea and hypoglycaemia, implicating impaired osmoreceptor function as the cause of his diabetes insipidus.

Both hypothermia and the SIADH are rare manifestations of neurological disease. The centres controlling thermoregulation and serum osmolality are both located within the hypothalamus. ${ }^{8,10}$ The organosum vasculosum of the lamina terminalis is thought to be the site of osmoreceptors, and the cells synthesizing ADH are located in the supraoptic and paraventricular nuclei. The pathways controlling thermoregulation are less well understood, but neurons that are responsive to temperature variation have been identified in the anterior hypothalamus and preoptic area. Al- $\overline{0}$ though we have no direct evidence, it is likely that $\frac{}{0}$ hypothalamic dysfunction in these areas is the $\frac{\bar{\sigma}}{\sigma}$ cause of our patient's chronic hypothermia and $\overparen{D}$ SIADH.

\title{
References
}

1. Sullivan, F., Hutchinson, M., Bahandeka, S. \& Moore, R.E. Chronic hypothermia in multiple sclerosis. J Neurol Neurosurg Psychiatry 1987, 50: 813-815.

2. Haak, H.R., van Hilten, J.J., Roos, R.A.C. \& Meinders, A.E. Functional hypothalamic derangement in a case of Wernicke's encephalopathy. Neth J Med 1990, 36: 291-296.

3. Lipton, J.M., Kirkpatrick, J. \& Rosenberg, R.N. Hypothermia and persisting capacity to develop fever. Occurence in a patient with sarcoidosis of the central nervous system. Arch Neurol 1977, 34: 498-504.

4. Ratcliffe, B.J., Bell, J.I., Collins, K.J., Frackowiak, R.S. \& Rudge, P. Late onset post-traumatic hypothermia. J Neurol Neurosurg Psychiatry 1983, 46: 72-74.

5. Nussey, S.S., Ang, V.T. \& Jenkins, J.S. Chronic hypernatraemia and hypothermia following subarachnoid haemorrhage. Postgrad Med J 1986, 62: 467-471.

6. Fox, R.H., Davies, T.W., Marsh, F.P. \& Urich, H. Hypothermia in a young man with an anterior hypothalamic lesion. Lancet 1970, 2: 185-188.

7. Bayliss, R.H. \& Thompson, C.J. Osmoregulation of vaso- 3 pressin secretion and thirst in health and disease. Clin Endocrinol 1988, 29: 549-576.

8. Lightman, S. Central nervous system control of fluid balance: $\infty$ physiology and pathology. Acta Neurochirurgica Suppl 1990, $\vec{\infty}$ 47: $90-94$.

9. Reuler, J.B. Hypothermia: pathophysiology, clinical setting and management. Ann Intern Med 1978, 89: 519-527.

10. Mitchell, D. \& Laburn, H.P. Pathophysiology of temperature regulation. Physiologist 1985, 28: 507-517.

\section{Spontaneous coronary artery dissection: a report of three cases and review of the literature}

\section{Peter Kearney, Harsh Singh², John Hutter ${ }^{2}$, Saleem Khan, Gary Lee and James Lucey ${ }^{1}$}

Department of Cardiology, University College Cork, South Infirmary Hospital, and ${ }^{1}$ Bon Secours Hospital, Cork, Ireland, and ${ }^{2}$ Bristol Royal Infirmary, Bristol, UK

Summary: We describe the clinical course of three patients who developed spontaneous coronary artery dissection. All patients were young women, one 9 weeks pregnant. All presented with chest pain; one died suddenly proving refractory to resuscitation, another developed unstable angina culminating in myocardial infarction, cardiogenic shock and death, and the third patient underwent coronary artery bypass grafting following diagnosis of a spontaneous coronary dissection of the left anterior descending artery at angiography. Pathological findings in the two fatal cases are reported.

This condition, although rare, is a prominent cause of ischaemic coronary events in young women, when it is frequently associated with pregnancy or the puerperium. Most patients die suddenly, but a clinical spectrum is seen including stable and unstable angina, myocardial infarction and cardiogenic shock. The left anterior descending artery is most frequently affected. The classical histological finding is that of a large haematoma occupying the outer third of the media resulting in complete compression of the true lumen.

The cause of spontaneous dissection remains unclear but theories of aetiology include a medial eosinophilic angiitis, pregnancy-induced degeneration of collagen in conjunction with the stresses of parturition, and rupture of the vasa vasorum. 
The diagnosis must be considered when a patient presents with a suggestive clinical profile. Urgent angiography should be undertaken to establish the diagnosis and consideration given to the need for coronary artery bypass grafting, which has been successfully employed in a number of patients. The uneventful long-term survival of cases treated conservatively has been reported.

\section{Introduction}

Spontaneous coronary dissection is a rare but increasingly reported cause of myocardial ischaemia and sudden cardiac death. It is seen commonly in young women and there is a strong association with the puerperium. A clinical spectrum is seen including stable and unstable angina, myocardial infarction, cardiogenic shock and frequently sudden death. Although the majority of cases are fatal, the diagnosis being made after death, there are a number of reports of successful medical and surgical intervention. The sporadic nature of the condition and small number of reported survivors make to difficult to draw firm conclusions as to the optimal forms of treatment. We report the course of three patients whose presentations exemplify the variable course the disorder may take and give rise to a number of therapeutic considerations.

\section{Case 1}

A previously well 34 year old farmer's wife was admitted to hospital complaining of central chest pain radiating to her face and both arms. The pain had begun 3 hours prior to arrival at the hospital, although she was pain free following admission. She had no known risk factor for ischaemic heart disease. An episode of unexplained chest and left arm pain had occurred some months before. Clinical examination was unremarkable. She was para 2, gravida 1 and was 9 weeks pregnant. Her electrocardiograph was normal. Within the first minutes of her admission, during her clinical examination, she developed ventricular fibrillation and subsequently a tonic-clonic seizure. Cardiopulmonary resuscitation was started but following an initially successful D.C. shock she developed refractory ventricular fibrillation and further resuscitation attempts were unsuccessful.

Postmortem examination revealed an area of dark, blueish discoloration at the junction of her middle and distal left anterior descending coronary artery. Sectioning revealed complete occlusion of the vessel at this point. Histology demonstrated medial dissection of the mid and distal left anterior descending artery with intramural thrombus accu-

Correspondence and present address: Peter Kearney, M.R.C.P.I., 2nd Medical Clinic, Johannes Gutenberg University, D-6500 Mainz, Germany.

Accepted: 12 May 1993 mulation and consequent compression of the vessel lumen. Patchy fibrosis of the distal septum was found but no evidence of acute myocardial infarction. Minimal atheroma was evident in both coronary arteries.

\section{Case 2}

A 31 year old woman, wheelchair bound with multiple sclerosis, presented to the accident and emergency department complaining of central chest pain of one hour's duration associated with breathlessness and sweating. She was a smoker of five cigarettes a day and had a family history of ischaemic heart disease. Her electrocardiograph was abnormal, demonstrating left axis deviation, lateral wall $T$ wave inversion and decreased $R$ wave amplitude in the chest leads. Despite the strongest advice, she refused hospital admission.

She returned on the evening of the next day experiencing persistent pain which worsened episodically. It now radiated to her left arm and breathlessness was more pronounced. Cardiovascular examination was unremarkable. Her cardiograph demonstrated septal $Q$ waves and $T$ wave inversion was more marked in V4-6. Creatine kinase was marginally elevated to twice the upper range of normal at $4.5 \mu \mathrm{kat} / \mathrm{l}$. She was admitted and treated for unstable angina with aspirin and infusions of heparin and glyceryl trinitrate, on which she initially stabilized. Thrombolysis was not administered. She later developed further severe chest pain and new anterolateral ST elevation was seen on her cardiograph. Her creatine kinase subsequently rose to $55 \mu \mathrm{kat} / 1$ and echocardiography demonstrated anteroapical akinesia. She developed refractory cardiogenic shock and congestive heart failure, and died on the seventh day of her admission.

A postmortem examination revealed grossly congested lungs. Extensive infarction of the left ventricle was evident. Sectioning revealed dissection of the proximal left main coronary artery which extended to the distal segments of both left anterior descending and left circumflex arteries. Minimal atheroma was found and no inflammatory process noted in the vessel wall. A serological screen for vasculitis, and anticardiolipin antibody and urinary aminoacid chromatography were negative. 


\section{Case 3}

A 37 year old farm hand was admitted to hospital with an anterior myocardial infarction. She had experienced exertional chest pain for the preceding 9 weeks, which she had largely ignored. She smoked 10 cigarettes a day and had a positive family history for ischaemic heart disease. She suffered from well-controlled coeliac disease and serum cholesterol was $3.8 \mathrm{mmol} / \mathrm{l}$. She was para 4 , gravida 4.

She developed postinfarction angina and underwent urgent coronary angiography. A dissection was seen at the junction of proximal and middle segments of the left anterior descending artery and the remainder of the coronary vasculature appeared free of disease (Figure 1). There was slow anterograde filling of the distal left anterior descending artery which was supported by collateral filling from the right coronary artery. Left ventriculography demonstrated apical dyskinesia and a hypokinetic free wall and septum. Coronary artery bypass grafting was performed, using the left internal mammary artery. The graft was inserted distal to the dissection and the proximal vessel was not tied off. She was discharged well and was pain free on subsequent follow-up.

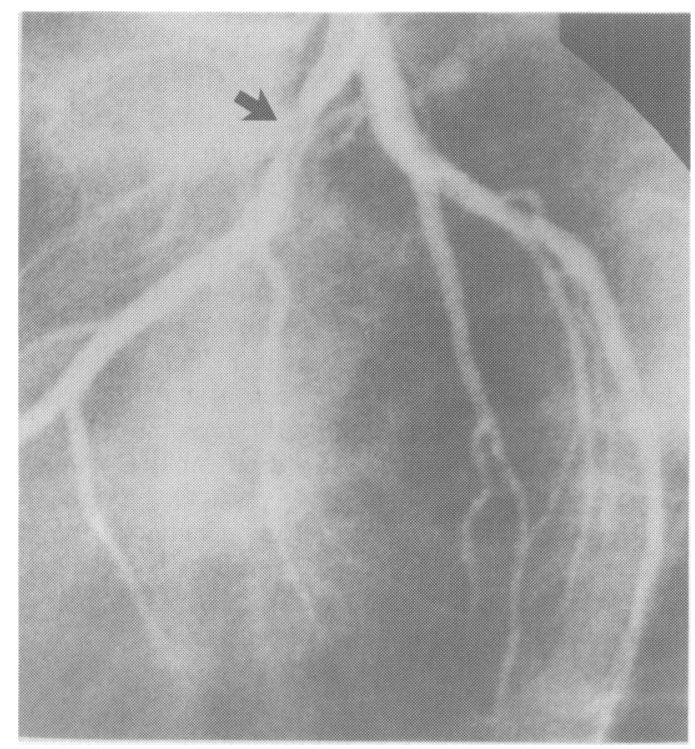

Figure 1 Angiogram of case 3, taken in the left anterior oblique projection, demonstrating a dissection in the proximal left anterior descending artery (arrow). The remainder of the coronary tree was free of disease.

\section{Discussion}

There have been $134^{1-10}$ cases of spontaneous coronary artery dissection reported in the world literature. Over two thirds of reported cases have been women and, of these, one third were either $\bar{O}$ pregnant (two previous cases) or in the puerperium (24 cases).

The left anterior descending artery is involved in $\overparen{\nabla}$ $80 \%$ of cases, the remainder involved the right coronary artery and a small number the left circumflex artery. There have been three reports of $\vec{\circ}$ involvement of both right and left coronary arteries $\overrightarrow{\vec{\omega}}$ and 19 reports of left main coronary involvement. Case 2 described above is the fourth report of a $\vec{\nabla}$ dissection extending from left main coronary artery to both left anterior descending and circumflex arteries. ${ }^{11-13}$

Coronary dissections may occur secondary to $\stackrel{\infty}{\infty}$ atherosclerosis, coronary angiography, percutan- $\dot{0}$ eous coronary interventions, bypass surgery, chest $\overrightarrow{0}$ trauma and Marfan's syndrome. Unlike aortic 을 dissections, no association with hypertension has been noted. Despite certain histological features found in a number of cases, the cause of 'primary' coronary dissection remains unknown and it is $\frac{\Phi}{3}$ improbable that a single mechanism operates in all $\underset{\mathbb{\Phi}}{\mathbb{Z}}$ patients.

Pathological examination reveals displacement $\frac{0}{0}$ distortion and usually complete compression of the lumen by a large haematoma (Figure 2). The plane of the dissection runs in the outer third of the media or between media and adventitia. Apart from these features, no uniform histological findings have been identified. An adventitial infiltrate of eosinophilic granulocytes was noted in eight patients studied by Robinowitz et al..$^{14}$ and, at the time of his review, a further 20 cases had been described in which similar infiltrates were evident. Cystic medial necrosis was evident in 17 patients in this series, but $\stackrel{-}{-}$ has been specifically excluded in a greater number. Intimal tears have been infrequently reported..$^{15,16} \dot{0}$

On the basis of his findings, Robinowitz postulated that spontaneous dissection results from an 8 accumulation of eosinophils which secrete lytic enzymes and major basic protein leading to medial 윽 weakening. A more recent report ${ }^{17}$ highlighted the case of a young man who died following myocardial rupture secondary to dissection and occlusion of a branch of his left circumflex artery. A marked $\sigma$ eosinophilic and mast cell infiltrate was found at $N$ the site of dissection. He had experienced a recent $\underset{\omega}{N}$ drug hypersensitivity reaction, which the authors 웅 felt may have induced an angiitis of his vasa 0 vasorum. A similar mechanism may have operated $\stackrel{\mathbb{D}}{\mathbb{D}}$ in a 70 year old man found to have dissection of an $\stackrel{?}{?}$ ectatic right coronary artery. He had a history of a 0 transient illness characterized by a marked eosinophilia and an associated cardiomyopathy. ${ }^{4}$ 


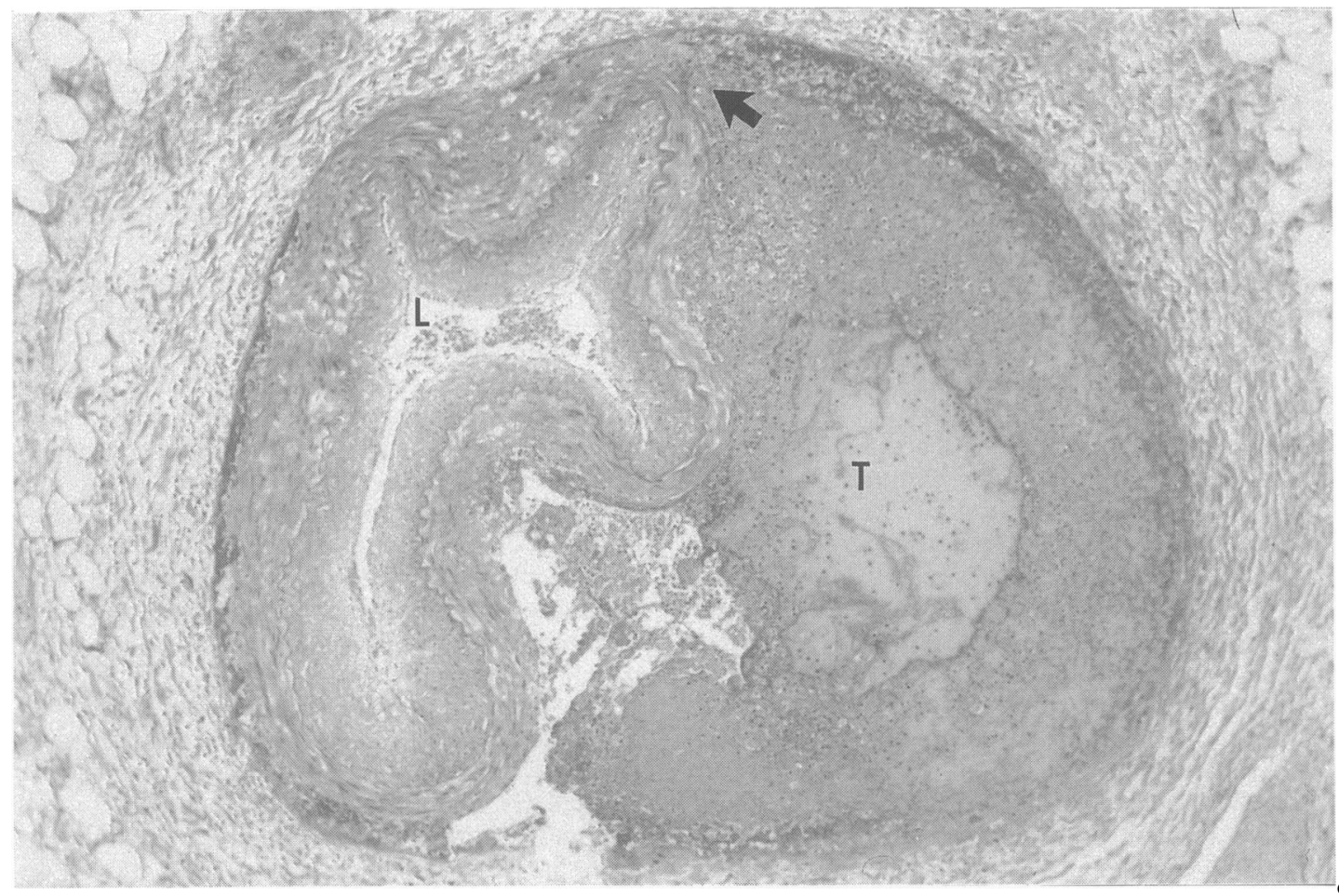

Figure 2 Histological section of a medial dissection found in the left anterior descending artery of case 2. Thrombus (T) occupies the outer third of the media and has obliterated the lumen $(\mathrm{L})$, which is almost completely compressed. An arrow points to the dissection plane running between media and adventitia.

Others emphasize the lack of any such findings in many autopsy reports and suggest that infiltrates, when present, are a secondary inflammatory phenomenon. ${ }^{18,19}$

It has been suggested that disruption of vasa vasorum may lead to intramedial haemorrhage and subsequent dissection. ${ }^{20}$ Although such vessels are more plentiful and fragile in the presence of developing atheroma ${ }^{21}$ they are scanty in the coronary circulation and are a relatively low pressure system, the rupture of which would seem unlikely to lead to luminal compression under normal circumstances. Normal coronaries may be more susceptible to luminal compression by intramedial haemorrhage in the absence of the stenting effect of atheroma, ${ }^{22}$ providing a potential explanation for the sex and age distribution of patients with this disorder.

Intimal tears might extend and lead to medial dissection. Although they are infrequently demonstrated, Claudon et al..$^{15}$ emphasize the meticulous technique that is required to outrule a tear with certainty, finding this in just one of 150 sections of the haematoma in the first of their patients. Angiographic diagnosis of coronary dissection depends on opacification of a false lumen which can occur only if a communication, as provided by a tear, exists. This lends support to the potential aetiological role of intimal disruption.

The degenerative effect of pregnancy on blood vessels, including the coronaries, in conjunction with increased blood flow and consequent intimal shear stress experienced at parturition has been suggested to underlie the concentration of cases in the puerperium..$^{23}$ Some support for this theory is found in a report of a 32 year old women who experienced anterior myocardial infarctions 2 and 4 months postpartum as a result of angiographically diagnosed primary coronary dissection. Fibroblasts were cultured from the patient and a reduction in the rate of total collagen synthesis relative to that of a control culture was seen, leading the authors to speculate on a disturbance of normal collagen synthesis as a result of hormonal changes in pregnancy and in the postpartum period. ${ }^{24}$

The majority of patients die before any intervention can be of benefit which may in part relate to the absence of any pre-existing stenoses and hence protective collaterals. In an early review of 43 cases, 
Smith $^{25}$ noted sudden death in $63 \%$ of cases and the proportion of cases dying suddenly remains largely unchanged since. The very low level of antemortem diagnosis may be explained by the high sudden death rate, although failure to include the condition in a differential diagnosis may also contribute. Recent reports highlight the good prognosis of those who survive the initial event; the asymptomatic survival of patients with spontaneous dissections who have been followed conservatively has been seen for up to 12 years. ${ }^{26}$ Spontaneous angiographic improvement has been documented in such a case. ${ }^{27}$

Behnam et al. ${ }^{2}$ report the successful administration of alteplase to a woman subsequently shown to have a primary dissection of her left anterior descending artery. They note a further four cases in which thrombolytic agents were given safely and suggest that luminal re-expansion can be established by lysing thrombus in the false lumen. We are aware of a more recent case who received successful thrombolysis on two occasions over a 4 year period for anterior myocardial infarctions prior to diagnosis of a large spontaneous dissection in her left anterior descending artery. Vacek et al.$^{28}$ administered intracoronary streptokinase in a case of iatrogenic coronary dissection with good effect.

When the patient survives the initial period, and remains unstable or suffers ongoing inducible ischaemia, coronary artery bypass grafting has been successful. ${ }^{20}$ It would appear to be unnecessary to ligate the vessel proximal to the graft anastomosis to prevent further dissection. Subsequent angiography may show evidence of residual dissection, despite successful revascularization. ${ }^{29}$ Internal mammary grafts are preferable, in view of the young age and otherwise excellent outlook of these patients. Percutaneous coronary balloon angioplasty has been successfully performed in a patient with primary dissection of the left anterior descending artery. ${ }^{30}$ There are two reports of

\section{References}

1. Yeoh, J., Choo, H., Soo, C., Lim, Y. \& Yan, C. Spontaneous coronary artery dissection in a young man with anterior myocardial infarction. Cathet Cardiovasc Diagn 1991, 24: 186-188.

2. Behnam, R. \& Tillinghast, S. Thrombolytic therapy in spontaneous coronary artery dissection. Clin Cardiol 1991, 14: 611-614.

3. Saunders, S. \& Ford, S. Primary coronary artery dissection possibly related to drug hypersensitivity in a male. Can $J$ Cardiol 1991, 7: 138-140.

4. Huikuri, H., Mallon, S. \& Myerburg, R. Cardiac arrest due to spontaneous coronary artery dissection in a patient with coronary ectasia - a case report. Angiology 1991, 42: $148-151$.

5. Fronc, H., MacMillan, R. \& Kimbiris, D. Coronary artery dissection - a case report. Angiology 1990, 41: 884-887.

6. Curiel, P., Spinelli, G., Petrella, A. et al. Postpartum coronary dissection followed by heart transplantation. Am J Obstet Gynecol 1990, 163: 538-539. patients receiving orthotopic cardiac transplants following primary dissection, one requiring the support of a Jarvik 7-70 artificial heart while a compatible heart was located. ${ }^{6,9}$

\section{Conclusions}

A number of important therapeutic issues are raised by the cases we report. It is possible that anticoagulation and anti-platelet therapy, both of proven benefit in unstable angina due to atheromatous coronary disease, might predispose to worsening of the dissection in a case of primary coronary dissection. This may explain the refractory unstable angina of case 2 and underlie the very extensive dissection found at autopsy. For similar reasons there is understandable reluctance to administer thrombolytic therapy in this condition, although a number of reports suggest that some patients have benefited.

The diagnosis must be considered in patients at low risk for atheroslcerotic heart disease who present with symptoms of myocardial ischaemia, particularly young women who are pregnant or in the puerperium. Urgent coronary angiography is necessary to make the diagnosis and should be undertaken when spontaneous coronary dissection is considered a possibility. If the patient remain unstable, medical therapy alone should not bôे relied upon and consideration should be given tơ urgent bypass grafting. Percutaneous coronary balloon angioplasty may have a role in certain cases. In those who remain stable, conservative management appears to be safe and compatible with an excellent long-term outlook.

\section{Acknowledgements}

We thank Dr W. Fennell, Dr H. Walsh and Dr N. Cahill for their contribution in the management of these cases.

7. Nishino, M., Kato, J., Exumi, A. et al. A case of primary coronary artery dissection. Kokyu To Junkan 1990, 38: 589-593.

8. Moor, D., Laska, J. \& Lindblom, D. Spontaneous coronary artery dissection treated by bypass surgery. Lakartidningen 1990, 87: 485-486.

9. Keon, W., Koshal, A., Boyd, W., Laramee, L., Farrell, E. \& Walley, V. Survival after spontaneous primary left main coronary artery dissection. Acute surgical intervention with the Jarvik 7-70 artificial heart. J Cardiovasc Surg 1989, 30: 786-789.

10. Lette, J., Gagnoon, A., Cerino, M. \& Prënovault, J. Apical hypertrophic cardiomyopathy with spontaneous post partum coronary artery dissection. Can J Cardiol 1989, 5: 311-314.

11. Razavi, M. Unusual forms of coronary artery disease. Cardiovasc Clin 1975, 7: 25-46.

12. Shaver, P., Carrig, T. \& Baker, W. Postpartum coronary artery dissection. $B r$ Heart $J$ 1978, 40: 83-86. 
13. Baker, P., Keyhani-Rofagha, S., Graham, R. \& Sharma, H. Dissecting hematoma (aneurysm) of coronary arteries. Am J Med 1986, 80: 317-319.

14. Robinowitz, M., Virmani, R. \& McAllister, H.A. Spontaneous coronary artery dissection and eosinophilic inflammation: a cause and effect relationship? Am J Med 1982, 72: 923-928.

15. Claudon, D., Claudon, D. \& Edwards, J. Primary dissecting aneurysm of coronary artery: a cause of acute myocardial ischemia. Circulation 1972, 45: 259-266.

16. Molloy, P., Ablett, M. \& Anderson, K. Left main stem coronary artery dissection. Br Heart $J$ 1980, 43: 705-708.

17. Saunders, S. \& Ford, S. Primary coronary artery dissection possibly related to drug hypersensitivity in a male. Can $J$ Cardiol 1991, 7: 138-140.

18. Sage, M., Koelmeyer, T. \& Smeeton, W. Fatal postpartum coronary artery dissection. A light and electron microscope study. Am J Forensic Med Path 1986, 7: 107-111.

19. Dowling, G. \& Buja, L. Spontaneous coronary artery dissection occurs with and without periadventitial inflammation. Arch Path Lab Med 1987, 111: 470-472.

20. Thayer, J., Healy, R. \& Maggs, P. Spontaneous coronary artery dissection. Ann Thorac Surg 1987, 44: 97-102.

21. Barger, A., Beeuwkes, R., Lainey, L. \& Silverman, K. Hypothesis: vasa vasorum and neovascularization of human coronary arteries: a possible role in the pathophysiology of atherosclerosis. $N$ Engl J Med 1984, 310: 175-177.
22. Bulkley, B. \& Roberts, W. Isolated coronary arterial dissection. J Thorac Cardiovasc Surg 1978, 67: 148-151.

23. Barrett, J., Van Hooydonk, J. \& Boehm, F. Pregnancy related rupture of arterial aneurysms. Obstet Gynecol Surv 1982, 37: 557-566.

24. Bonnet, J., Aumailley, M., Thomas, D. et al. Spontaneous coronary artery dissection: case report and evidence for a defect in collagen metabolism. Eur Heart J 1986, 7: 904-909.

25. Smith, J. Dissection aneurysms of coronary arteries. Arch Pathol 1975, 99: 117-121.

26. DeMaio, S., Kinsella, S. \& Silverman, M. Clinical course and long-term prognosis of spontaneous coronary artery dissection. Am J Cardiol 1989, 64: 471-474.

27. Werner, G.S., Wojcik, J., Wiegand, V. \& Kreuzer, H. Spontaneous coronary artery dissection: a rare cause of myocardial infarct. Z Kardiol 1990, 79: 32-36.

28. Vacek, J. \& McKiernan, T. Intracoronary streptokinase for acute coronary artery dissection. $N$ Engl J Med 1984, 310: 1187.

29. Matsuura, A., Yasuura, K., Sawasaki, M. et al. Spontaneous coronary artery dissection: a case report and a review of the literature. Kyoba Geka 1992, 45: 243-246.

30. Gonzales, J., Hill, J. \& Conti, C. Spontaneous coronary artery dissection treated with percutaneous transluminal angioplasty. Am J Cardiol 1989, 63: 885-886.

\title{
Successful use of a transjugular intrahepatic portosystemic stent shunt to control severe refractory oesophageal variceal haemorrhage in a poor risk patient
}

\author{
I.L.P. Beales, J.E. Jackson ${ }^{1}$, M. Rudolf and J. Arnold
}

Department of Medicine, Ealing Hospital NHS Trust, Uxbridge Road, Southall, Middlesex UB1 3HW and 'Department of Diagnostic Radiology, Royal Postgraduate Medical School, Hammersmith Hospital,

Du Cane Road, London W12 ONN, UK

\begin{abstract}
Summary: This report describes a 44 year old man with a severe gastrointestinal haemorrhage from oesophageal varices. Bleeding could not be controlled with conservative therapy and sclerotherapy. He was successfully treated with a radiologically guided transjugular intrahepatic stent shunt at a time when his condition was too poor to attempt an open surgical procedure.
\end{abstract}

\section{Introduction}

Haemorrhage from oesophageal varices in patients with portal hypertension remains a common and serious medical emergency associated with significant mortality and morbidity. Injection sclerotherapy is the most effective primary therapy for acute variceal bleeding. Pharmacotherapy and

Correspondence and present address: I.L.P. Beales, B.Sc., M.R.C.P., Department of Gastroenterology, Hammersmith Hospital, Du Cane Road, London W12 0NN.

Accepted: 17 May 1993 balloon tamponade have useful supporting roles. ${ }^{1,2}$ Despite therapy, some $10-20 \%$ of patients may continue bleeding ${ }^{3-5}$ and in these cases the prognosis is poor. ${ }^{3}$ We report a case of severe refractory bleeding in a patient with poor outlook, eventually controlled with a transjugular intrahepatic portosystemic stent shunt (TIPSS). This should lead to wider consideration and evaluation of this technique as an alternative to surgery in critically ill patients. 